\title{
Large Deviation Principle for the Empirical Degree Measure of Preferential Attachment Random Graphs
}

\author{
K. Doku-Amponsah ${ }^{1}$, F. O. Mettle ${ }^{1} \&$ E. N. N. Nortey ${ }^{1}$ \\ ${ }^{1}$ School of Physical and Mathematical Sciences, College of Basic and Applied Sciences,University of Ghana, \\ Legon, Ghana \\ Correspondence: K. Doku-Amponsah, School of Physical and Mathematical Sciences, College of Basic and Ap- \\ plied Sciences,University of Ghana, Legon, Ghana. Tel: 233-205-164-254. E-mail: kdoku@ug.edu.gh
}

Received: April 4, 2014 Accepted: April 28, 2014 Online Published: January 8, 2015

doi:10.5539/ijsp.v4n1p76 URL: http://dx.doi.org/10.5539/ijsp.v4n1p76

\begin{abstract}
We consider preferential attachment random graphs which may be obtained as follows: It starts with a single node. If a new node appears, it is linked by an edge to one or more existing node(s) with a probability proportional to function of their degree. For a class of linear preferential attachment random graphs we find a large deviation principle (LDP) for the empirical degree measure. In the course of the prove this LDP we establish an LDP for the empirical degree and pair distribution see Theorem 0.3, of the fitness preferential attachment model of random graphs.
\end{abstract}

Keywords: Large deviation principle, preferential attachment graphs, empirical degree measure, path empirical degree measure

Mathematics Subject Classification: 60F10, 05C80

\section{Introduction}

Preferential attachment (P.A) random graph models have become extremely popular in the last two decades since they were first studied by (Barabasi and Albert ,1999). Example (Van der Hofstad ,2013), (Newman, 2003) and (Newman et. al, 2006) provide good overviews.

The P.A model of random graphs are graphs in which nodes are added sequentially and attach to exactly one randomly chosen existing node and the chance a new node connects to an existing node is proportional to its degree.

The model is typically generalized to allow for vertices to have $m>1$ initial edges by collapsing $m$ vertices in the one initial edge case into a single vertex (possibly causing loops). The most studied feature of these objects is the distribution of the degrees of the nodes; that is, the proportion of nodes that have degree $k$ as the graph grows large. See, example (Collevecchio et. al, 2013), (Krapivsky et. al, 2000), (Rudas et. al, 2007) for results on more general attachment rules.

Few large deviation results for P.A model have so far been found. In paper ( Choi et. al, 2011), P.A schemes where the selection mechanism is possibly time-dependent are considered, and an in infinite dimensional large deviation principle for the sample path evolution of the empirical degree distribution is found by Dupuis-Ellis type methods.

(Dereich and Moerters, 2009) studied a dynamic model of random networks, where new vertices are connected to old ones with a probability proportional to a sub-linear function of their degree. For this model of random networks, they obtained a strong limit law for the empirical degree distribution. Results on the temporal evolution of the degrees of individual vertices via large and moderate deviation principles were also found.

(Bryc et. al, 2009) found the large deviation principle and related results for a class of Markov chains associated to the 'leaves' in P.A model of random graphs using both analytic and Dupuis-Ellis-type path arguments. Recently, (Doku-Amponsah et. al, 2014) proved a large deviation upper bound for fitness preferential attachment random network.

In this paper, we find a large deviation principle for the empirical degree distribution of preferential attachment 
random network in the linear regime. See, Theorem 0.1. In the course of the proof of Theorem 0.1 , we find a large deviation principle for the empirical degree and pair measure of the fitness preferential attachment random networks, see Theorem 0.2 and a joint LDP for the empirical degree and pair measure, and the sample path empirical degree distribution of the fitness preferential attachment random networks, see Theorem 0.3. The main technique in our proof is exponential change of measure, see example (Doku-Amponsah et. al, 2014) and the method of mixtures, see (Biggins, 2004).

\section{Main Results}

\subsection{LDP for the Preferential Attachment Model of Random Graphs}

Let $f:\{0,1,2, \ldots\} \rightarrow[0, \infty]$ be a weight function. We define a preferential attachment random graph as follows: It starts with single vertex serving as root. If a new vertex $n$ is introduced, it connects to vertices $v_{n} \in\{1, \ldots, n-1\}$ independently with probability proportional to $f\left(N\left(v_{n}\right)\right)$, where $N(m)$ is the in-degree of vertex $m$.

We write $\mathcal{N}=\{0,1,2, \ldots\}$. In this paper, we shall restrict ourself to functions of the form

$$
f(k)=\gamma k+\beta, \text { where } \gamma, \beta \in(0, \infty]
$$

We define empirical degree measure $\mathcal{L}$ on $\mathcal{N}$ by

$$
\mathcal{L}(k)=\frac{1}{n-1} \sum_{m=1}^{n-1} \delta_{N^{(m)}\left(j_{m}\right)}(k) .
$$

We denote by $\mathcal{M}(\mathcal{N})$ the space of probability measures on $\mathcal{N}$, equipped with the topology generated by total variation metric $\|\pi-\hat{\pi}\|:=\frac{1}{2} \sum_{k=0}^{\infty}\|\pi(k)-\hat{\pi}(k)\|$.

Theorem 0.1. Suppose $X$ is $P$.A random graph with linear weight function $f: \mathcal{N} \rightarrow[0, \infty]$, satisfying $\gamma \geq 1-\beta$, $\log (1+\beta / \gamma)<\infty$ and

$$
\sum_{k=0}^{\infty} \frac{1}{\gamma k+\beta}=\infty .
$$

Then, as $n \rightarrow \infty$, the empirical degree measure $\mathcal{L}$, satisfies a large deviation principle in $\mathcal{M}(\mathcal{N})$ with good rate function

$$
I(\ell)=H\left(\ell \| \frac{(\gamma+\beta)}{f} \otimes \hat{\ell}\right),
$$

where $\frac{(\gamma+\beta)}{f} \otimes \hat{\ell}(k)=\frac{(\gamma+\beta)}{f(k)} \hat{\ell}(k)$ and $\hat{\ell}(k)=11-\sum_{j=0}^{k} \ell(k)$.

\subsection{Large-deviations for Fitness P.A Random Network}

To establish Theorem 0.1 we pass to a more general random preferential random graph, the fitness or coloured preferential random graph. We write $\mathcal{N}=\mathbb{N} \cup\{0\}$. Given a weight function $f_{m / n}: \mathcal{N} \times \mathcal{X} \rightarrow[0, \infty], m=1,2,3, \ldots n$ and a probability law $\mu$ on finite alphabet $\mathcal{X}$, we define coloured (fitness) P.A random network with $n$ vertices as follows:

- Assign vertex $m=1$ (the root of the network) colour $X(m)$ according to $\mu: \mathcal{X} \rightarrow[0,1]$.

- If a new vertex $m$ is introduced, it gets colour $X(m)$ independently according $\mu$,

- it connects to vertices $v_{m} \in\{1, \ldots, m-1\}$ independently with probability proportional to

$$
f_{m / n}\left(N\left(v_{m}\right), A(m)\right)
$$

where $A(m)=\left(X\left(v_{m}\right), X(m)\right)$ and $N(m)$ is the in-degree of vertex $m$.

- Repeat the previous three steps until we have $n$ vertices.

We consider $\left\{\left(N\left(v_{m}\right), A(m)\right): m=1,2,3, \ldots, n \ldots\right\}$ under the joint law of colour and tree. Denote by $X$ a typed tree and by $X(i)$ colour of vertex $i$. We write $\mathcal{X}^{*}=\mathcal{X} \times \mathcal{X}$. In this paper, we shall restrict ourself to functions of the form

$$
f_{t}(k, a)=\gamma(t, a) k+\beta(t, a)
$$


where $\gamma:(0,1] \times \mathcal{X}^{*} \rightarrow(0, \infty], \beta:(0,1] \times \mathcal{X}^{*} \rightarrow[0, \infty]$. We assume

$$
\gamma(t, a)+\beta(t, a):=c_{t}, \text { for all }(t, a) \in(0,1] \times \mathcal{X} .
$$

Let $N^{(m)}(i)$ be the degree of vertex $i$ at time $m$ and observe that at time $n$, the law of the fitness P.A graph is given by

$$
\mathbb{P}_{f}^{(n)}(X)=\prod_{m=1}^{n} \mu(X(m)) \times \prod_{m=2}^{n} \frac{f_{m / n}\left(N^{(m)}\left(j_{m}\right), A(m)\right)}{\sum_{i=1}^{m-1} f_{m / n}\left(N^{(m)}(i), A(m)\right) .}
$$

For every $X$, we define empirical degree and pair measure measure $M_{X}$ on $\mathcal{N} \times \mathcal{X}^{*}$ by

$$
M_{X}(k, a)=\frac{1}{n-1} \sum_{m=1}^{n-1} \delta_{\left(N^{(m)}\left(j_{m}\right), A(m)\right)}(k, a) .
$$

We write $\ell_{m}(a)=\left\{j_{m} \in\{1,2,3, \ldots, m-1\}: x\left(j_{m}\right)=a_{1}, x(m)=a_{2}\right\}$ and for every $m=2,3,4, \ldots, n-1$ we define a probability measure on $\mathcal{N} \times \mathcal{X}^{*}$ by

$$
L_{\frac{m}{n}}^{X}(k, a)=\frac{1}{m-1} \sum_{j=1}^{m-1} \delta_{N^{(m)}(j)}(k) \mathbb{1}_{\left\{j \in \ell_{m}(A(m)\}\right.} \otimes \delta_{A(m)}(a),
$$

where

$$
\mathbb{1}_{\left\{j \in \ell_{m}(b)\right\}} \otimes \delta_{b}(a)= \begin{cases}\mathbb{1}_{\left\{j \in \ell_{m}(b)\right\}} & \text { if } b=a, \\ 0 & \text { otherwise. }\end{cases}
$$

and notice,

$$
L_{1}^{X}(k, a)=M_{X}(k, a)
$$

We denote by $\mathcal{M}(\mathcal{X})$ the space of probability measures on $\mathcal{X}$ equipped with the weak topology and $\mathcal{M}\left(\mathcal{N} \times \mathcal{X}^{*}\right)$ the space of probability measures on $\mathcal{N} \times \mathcal{X}^{*}$, equipped with the topology generated by total variation metric.

$$
\|\pi-\hat{\pi}\|=\frac{1}{2} \sum_{(k, a) \in \mathcal{N} \times X^{*}}\|\pi(k, a)-\hat{\pi}(k, a)\| .
$$

Let $\lim _{n \rightarrow \infty} L_{[n t] / n}^{X}=v_{t}$ and write $v:=\left(v_{t}, t \in[0,1]\right)$. The following is an $\operatorname{LDP}$ for $\left(M_{X},\left(L_{[n t] / n}^{X}, t \in[0,1]\right)\right)$.

Theorem 0.2. Suppose $X$ is coloured P.A random graph with colour law $\mu: X \rightarrow(0,1]$ and linear weight functions $\left(f_{t}, t \in(0,1]\right)$ satisfying $\inf _{t \in(0,1]} c_{t} \geq 1$,

$$
\sup _{a \in \mathcal{X}^{*}} \int_{0}^{1} \log (1+\beta(t, a) / \gamma(t, a)) d t<\infty
$$

and

$$
\inf _{(t, a) \in(0,1] \times X^{*}} \sum_{k=0}^{\infty} \frac{1}{\gamma(t, a) k+\beta(t, a)}=\infty .
$$

Then, as $n \rightarrow \infty$, the pair of empirical measures $\left(M_{X},\left(L_{[n t] / n}^{X}, t \in[0,1]\right)\right)$ satisfies a large deviation principle in $\mathcal{M}\left(\mathcal{N} \times \mathcal{X}^{*}\right) \times\{v\}$ with good rate function

$$
\tilde{J}(\omega, v)= \begin{cases}\left.H\left(\omega_{2,1} \| \mu\right)\right)+\sum_{a \in X} \omega_{2}(a) \int_{[0,1]} H\left(\omega(\cdot \mid a) \| \frac{c_{t}}{f_{t}} \otimes v_{t}(\cdot \mid a)\right) d t, & \text { if } \omega=v_{1}, \\ \infty & \text { otherwise, }\end{cases}
$$

where $\omega_{2,1}$ is the $\mathcal{X}$ - marginal of the probability measure $\omega_{2}$ and

$$
\frac{c_{t}}{f_{t}(\cdot, a)} \otimes v_{t}(\cdot \mid a)(k)=\frac{c_{t}}{f_{t}(k, a)} v_{t}(k \mid a) .
$$

Our next theorem which generalizes Theorem 0.1 is a special case of Theorem 0.2 above. 
Theorem 0.3. Suppose $X$ is coloured P.A random graph with colour law $\mu: \mathcal{X} \rightarrow(0,1]$ and linear weight function $f: \mathcal{N} \times \mathcal{X}^{*} \rightarrow[0, \infty]$ satisfying $c \geq 1$,

$$
\sup _{a \in \mathcal{X}^{*}} \log (1+\beta(a) / \gamma(a))<\infty
$$

and

$$
\inf _{a \in \mathcal{X}^{*}} \sum_{k=0}^{\infty} \frac{1}{\gamma(a) k+\beta(a)}=\infty
$$

Then, as $n \rightarrow \infty, M_{X}$ satisfies a large deviation principle in $\mathcal{M}\left(\mathcal{N} \times \mathcal{X}^{*}\right)$ with good rate function

$$
\left.J(\omega)=H\left(\omega_{2,1} \| \mu\right)\right)+\sum_{a \in \mathcal{X}} \omega_{2}(a) H\left(\omega(\cdot \mid a) \| \frac{c}{f} \otimes \hat{\omega}(k \mid a)\right)
$$

where $\hat{\omega}(k \mid a):=\mathbb{1}-\sum_{j=0}^{k} \omega(k \mid a)$.

Observe that $J(\omega)=0$ if and only if $\omega(k, a)=\frac{c \omega_{2}(a)}{f(k, a)}\left(\mathbb{1}-\sum_{j=0}^{k} \omega(k \mid a)\right)$, and hence solving recursively for $\omega(\cdot \mid a)$ we get

$$
\omega(k \mid a)=\pi_{f}(k \mid a):=\frac{c}{c+f(k, a)} \prod_{i=0}^{k-1} \frac{f(i, a)}{c+f(i, a)} .
$$

Here we remark that conditions $(0.1)$ and $(0.3)$ are necessary for $\pi_{f}(\cdot \mid a)$ to be a probability measure on $\mathcal{N}$. See (Dereich and Morters, 2009, p. 13). Note, if $f(k, a)=w(k)$ then (0.4) concise with the asymptotic degree distribution of random trees and general branching processes found in (Rudas et. al, 2008).

\section{Proof of Results}

\subsection{Dynamics of the Path Empirical Degree Distribution}

Denote by $\mathcal{D}([0,1], \mathbb{R})$ the space of right continuous left limited(cadlag) paths from $[0,1]$ to $\mathbb{R}$. We define the sample path space

$$
\begin{aligned}
& \mathcal{D}_{\mathcal{M}}:=D([0,1]: \mathcal{M}(\mathcal{N} \times \mathcal{X})) \\
& =\{\text { the set of all } v:[0,1] \mapsto \mathcal{M}(\mathcal{N} \times \mathcal{X}) \text { such that } v(k, a) \in \mathcal{D}([0,1], \mathbb{R}) \text { for all } k \geq 0, a \in \mathcal{X} \text { and }\langle v\rangle=1\}
\end{aligned}
$$

and endow it with the topology of uniform convergence associated with the norm

$$
\|v-\hat{v}\|:=\sup _{t \in[0,1]}\left\|v_{t}-\hat{v}_{t}\right\| .
$$

For any $v \in \mathcal{D}_{\mathcal{M}}$ we write $v_{t}(k \mid a):=\frac{v_{t}(k, a)}{\sum_{k=0}^{\infty} v_{t}(k, a)}$, for all $t \in[0,1]$ and $(k, a) \in \mathcal{N} \times \mathcal{X}$. Write $\dot{v}_{t}:=\frac{d v_{t}}{d t}$ for the time derivative of the measure $v_{t}$ and we associate with each path $v \in \mathcal{D}_{\mathcal{M}}$ the relaxed measure on $[0,1] \times(\mathcal{N} \times \mathcal{X})$

$$
\bar{v}(d k, d t \mid a)=v_{t}(d k \mid a) d t .
$$

We call $v \in \mathcal{D}_{\mathcal{M}}$ absolutely continuous if for each $k \in \mathbb{N}$, there exists $\dot{v}(k \mid a)$ such that

$$
v_{1}(k \mid a)-v_{0}(k \mid a)=\int_{0}^{1} \dot{v}_{s}(k \mid a) d s .
$$

For each absolutely continuous path $v$, we define $v^{v}(\cdot \mid a), \bar{v}(\cdot, \cdot \mid a)$ - almost everywhere by

$$
v_{t}^{v}(k \mid a):=-\sum_{i=0}^{k} \dot{v}_{t}(i \mid a) .
$$

By $v^{v} \ll v$ we mean $v$ is absolutely continuous. We write

$$
\mathcal{D}_{\mathcal{M}_{n}(\mathcal{N} \times X)}:=\left\{v \in \mathcal{D}_{\mathcal{M}(\mathcal{N} \times X)}:([n t]-1) v_{[n t] / n} \in \mathbb{N}, \forall t \in[0,1)\right\} .
$$


Note that the measure $L_{[n t] / n}^{X}$, for $t \in[0,1)$ is deterministic and its distribution is degenerate at some $v_{[n t] / n}$, for $t \in[0,1)$ converging to $v_{t}, t \in[0,1)$.

\subsection{Exponential Change-of-Measure}

Throughout the remaining part of this paper, we assume the sample path degree distribution $v$ satisfies $v_{t}(k \mid a)=$ $v_{t}^{v}(k \mid a)$, for all $t \in[0,1]$.

Let $\tilde{g}: \mathbb{N} \times \mathcal{X} \rightarrow \mathbb{R}$, and write $\lim _{n \rightarrow \infty} L_{\frac{[n t]}{n}}:=v_{t} \in \mathcal{D}_{\mathcal{M}}$, we define the function $U_{\tilde{g}}:[0,1] \times \mathcal{X} \rightarrow \mathbb{R}$ by

$$
U_{\tilde{g}}^{(n)} \otimes v(a)=\log \left\langle\frac{e^{\tilde{s}[n t] / n}(;, a)}{f_{[n t] / n}(\cdot a)}, v_{\frac{[n t]}{n}}(\cdot \mid a)\right\rangle,
$$

and note that

$$
\lim _{n \rightarrow \infty} U_{\tilde{g}_{t}}^{(n)} \otimes v(a)=\log \left\langle\frac{e^{\tilde{s}_{t}(t, a)}}{f_{t}(\cdot, a)}, v_{t}(\cdot \mid a)\right\rangle=: U_{\tilde{g}_{t}} \otimes v(a, t) .
$$

We use $\tilde{g}$ to define a new fitness P.A random graph with $n$ vertices as follows:

- At time $m=$ assign the root $m$ of the network fit $X(m)$ according to the law $\tilde{\mu}$ given by

$$
\tilde{\mu}\left(a_{1}\right)=e^{\tilde{h}\left(a_{1}\right)-U(\tilde{h})} \mu\left(a_{1}\right) .
$$

- For any other time $m$ new node $m$ which appear gets fit $X(m)$ according to the fit law $\tilde{\mu}$. It connects to node $v_{m}$, independently with probability proportional to

$$
\tilde{f}_{m / n}\left(N^{(m)}\left(v_{m}\right), A(m)\right)=\frac{c_{m / n}}{f_{m / n}\left(N^{(m)}\left(v_{m}\right), A(m)\right)} e^{\tilde{g}_{m / n}\left(N^{(m)}\left(v_{m}\right), A(m)\right)} .
$$

- Repeat the previous three steps until we have $n$ vertices.

We denote by $\mathbb{P}_{\tilde{f}, n}$ the law of the new fitness P.A graph and observe that it is absolute continuous with respect to $\mathbb{P}_{f, n}$, as for fitness graph $X$ we have that

$$
\begin{aligned}
& \frac{d \mathbb{P}_{\tilde{f}, n}}{d \mathbb{P}_{f, n}}(X)=\prod_{m=1}^{n} \frac{\tilde{\mu}(X(m)}{\mu(X(m)} \times \frac{\prod_{m=1}^{n-1} \tilde{f}_{m / n}\left(N^{(m)}\left(j_{m}\right), A(m)\right)}{\prod_{m=2}^{n-1} \sum_{i=1}^{m-1} \tilde{f}_{m / n}\left(N^{(m)}(i), A(m)\right)} \times \frac{\prod_{m=2}^{n-1} \sum_{i=1}^{m-1} f_{m / n}\left(N^{(m)}(i), A(m)\right)}{\prod_{m=1}^{n-1} f_{m / n}\left(N^{(m)}\left(j_{m}\right), A(m)\right)} \\
& \left.=e^{(n-1)\left\langle\tilde{h}-U(\tilde{h}), M_{X}\right\rangle+(n-1)\left\langle\tilde{g}_{\cdot / n}-2 \log f_{\cdot / n}+\log c, M_{X} \otimes i d\right.}\right\rangle-(n-1)\left\langle U_{\tilde{s} \cdot / n} \otimes L, M_{X} \otimes i d\right\rangle,
\end{aligned}
$$

where $i d$ is the identity function from $[0,1]$ to $[0,1]$. The following Lemma will be used to establish the upper bound in a variational formulation.

Lemma 0.4. For every $\theta>0$ there exits a compact set $K_{\theta} \subset \mathcal{M}\left(X^{*}\right)$ such that

$$
\limsup _{n \rightarrow \infty} \frac{1}{n} \log \mathbb{P}_{f, n}\left\{M_{X} \notin K \mid\left(L_{[n t]] / n}=v_{[n t] / n, \forall t \in(0,1]}\right)\right\} \leq-\theta .
$$

Proof. Let $1 \geq \delta>0$, and $l \in N$. We choose $k(l, \delta) \in \mathbb{N}$ large enough such that, for large $n$, we have

$$
\sum_{i=1}^{[n t]-1} e^{l\left[\mathbb{1}_{[N([n t)(i)>k(l, \delta)}\right.} \frac{\left.f_{(n n] / n}\left(N^{(n n t)}\right)(i), a\right)}{c([n t]-1)} \leq 2 e^{\delta}, \text { for all } a \in \mathcal{X} \text { and for all } t
$$


Now using Chebyschev's inequality we have

$$
\begin{aligned}
& \mathbb{P}_{f, n}\left\{M_{X}\left(N^{[[n t])}>k(l, \delta)\right) \geq l^{-1}, L_{[n t] / n}=v_{[n t]] / n, \forall t \in(0,1]}\right\} \\
& \leq e^{-n l} \mathbb{E}\left\{e^{\sum_{m=1}^{n-1} l^{2} \mathbb{U}_{\left(N N^{(m)}\left(j_{m}\right)>k(l, D)\right.}}, L_{\frac{m}{n}}=v_{\frac{m}{n}}, m=2,3,4, \ldots, n-1\right\} \\
& =e^{-n l} \prod_{m=2}^{n} \mathbb{E}\left\{e^{l^{2} \mathbb{I}_{(\mathbb{N}(m)}(j m)>k(l, \delta) \mid}, L_{\frac{m}{n}}=v_{\frac{m}{n}}\right\} \\
& \leq e^{-n l}\left[\sup _{a \in \mathcal{X}} \sup _{t \geq 0}\left(\sum_{i=1}^{[n t]-1} e^{l^{2}} \mathbb{1}_{\left\{N^{(n n t)}(i)>k(l, \delta)\right\}} \frac{f_{[n t] / n}\left(N^{([n t)}(i), a\right)}{([n t]-1)\left\langle\left\langle f_{[n t] / n}, \frac{\underline{n}}{\frac{[n t]}{n}}(\cdot \mid a)\right\rangle\right.}\right)\right]^{n} \\
& =e^{-n l}\left[\sup _{a \in X} \sup _{t \geq 0}\left(\sum_{i=1}^{[n t]-1} e^{l^{2}} \mathbb{1}_{\left\{N^{([n t)}(i)>k(l, \delta)\right\}} \frac{f_{[n t] / n}\left(N^{([n t])}(i), a\right)}{c([n t]-1)}\right)\right]^{n} \\
& \leq e^{-n l} \times\left(2 e^{\delta}\right)^{n} \\
& =e^{n(l-\delta-\log 2)}
\end{aligned}
$$

Now given $\theta$ we choose $M>\theta+\delta+\log 2$ and define the set

$$
\Gamma_{\delta, \theta}:=\left\{v: v(N>k(l, \delta))<l^{-1}, l \geq M\right\}
$$

As $\{N \leq k(l, \delta)\}$ is pre-compact, $\Gamma_{\delta}$ is compact in the weak topology by prokohov criterion. Moreover

$$
\mathbb{P}_{f, n}\left\{M_{X} \notin K_{\theta} \mid\left(L_{[n t] / n}=v_{[n t] / n, \forall t \in(0,1]}\right)\right\} \leq \frac{1}{1-e^{-1}} \frac{e^{-\theta}}{\mathbb{P}\left\{L_{[n t] / n}=v_{[n t] / n, \forall \in[0,1]}\right\}}=\frac{1}{1-e^{-1}} e^{-\theta} .
$$

Now letting $K_{\theta}$ be the closure of $\cap_{1 \geq \delta>0} \Gamma_{\delta, \theta}$ and taking limit as $n$ approaches $\infty$ we have (0.7) which ends the proof the Lemma.

\subsection{Proof of Theorem 0.2.}

We derive the upper bound in a variational formulation. To do this, we denote by $C_{1}$ the space of all functions on $\mathcal{X}$ and by $C_{2}$ the space of all bounded continuous functions on $\mathcal{N} \times \mathcal{X}^{*}$. We define on the space of probability measures $\mathcal{M}(\mathcal{N} \times \mathcal{X})$ the function $\hat{K}$ given by

$$
\begin{aligned}
\hat{K}_{v}(\omega)=\int_{[0,1]} \sup _{\tilde{g} \in C_{2}, \tilde{h} \in C_{1}}\left\{\int(\tilde{h}-U(\tilde{h})) \omega_{2,1}\left(d a_{1}\right)\right. & +\int \tilde{g}_{t}(k, a) \omega(d k, d a)-2 \int \log \tilde{f}_{t}(k, a) \omega(d k, d a) \\
& \left.+\log c_{t}-\int U_{\tilde{g}_{t}} \otimes v(a, t) \omega_{2}(d a)\right\} d t .
\end{aligned}
$$

Lemma 0.5. For every close set $F \subset \mathcal{M}(\mathcal{N} \times \mathcal{X})$ we have

$$
\limsup _{n \rightarrow \infty} \frac{1}{n} \log \mathbb{P}_{f, n}\left\{M_{X} \in F \mid\left(L_{[n t] / n}=v_{[n t] / n, \forall t \in(0,1]}\right)\right\} \leq-\inf _{\omega \in F} \hat{K}_{v}(\omega)
$$

Proof. We let $\tilde{h} \in C_{1}, \tilde{g} \in C_{2}$ and use the Jensen's inequality to obtain

$$
\begin{aligned}
& e^{\left(\sup _{a_{1}} \tilde{h}(a)-\inf _{a_{1}} \tilde{h}\left(a_{1}\right)\right)} \leq \int e^{\tilde{h}(X(n))-U(\tilde{h})} d \tilde{\mathbb{P}}_{f, n} \\
& =\mathbb{E}\left\{e^{(n-1)}\left[\left\langle\tilde{h}-U(\tilde{h}), M_{X}\right\rangle+\left\langle\tilde{g}_{[n t] / n}-2 \log f_{[n t] / n}+\log c_{t}, M_{X} \otimes i d\right\rangle-\left\langle U_{\tilde{s}_{[n t] / n}} \otimes L, M_{X} \otimes i d\right\rangle\right],\left(L_{[n t] / n}=v_{[n t] / n, \forall t \in(0,1]}\right)\right\} .
\end{aligned}
$$

This yields the inequality

$$
\begin{gathered}
\limsup _{n \rightarrow \infty} \frac{1}{n} \log \mathbb{E}\left\{e^{(n-1)}\left[\left\langle\tilde{h}-U(\tilde{h}), M_{X}\right\rangle+\left\langle\tilde{g}_{[n t] / n}-2 \log f_{[n t] / n}+\log c_{t}, M_{X} \otimes i d\right\rangle-\left\langle U_{\tilde{s}[n] / n} \otimes L, M_{X} \otimes i d\right\rangle\right]\right. \\
\left.\mid\left(L_{[n t] / n}=v_{[n t] / n, \forall t \in(0,1]}\right)\right\}=0 .
\end{gathered}
$$


Given $\varepsilon>0$, define $\hat{K}_{\varepsilon, v}$ by $\hat{K}_{v, \varepsilon}(\omega)=\min \left\{\hat{K}_{v}(\omega), \varepsilon^{-1}\right\}-\varepsilon$. For $\omega \in F$ we fix $\tilde{h} \in C_{1}$ and $\tilde{g} \in C_{2}$ such that

$$
\left\langle\tilde{h}-U(\tilde{h}), \omega_{2,1}\right\rangle+\left\langle\tilde{g}_{t}-2 \log f_{t}+\log c_{t}, \omega \otimes i d\right\rangle-\left\langle U_{\tilde{g}_{t}}^{v}, \omega \otimes i d\right\rangle \geq \hat{K}_{r, \varepsilon}(\omega) .
$$

Now, because the function $\tilde{g}_{t}$ is bounded, we can find open neighbourhood $B_{\omega}$ of $\omega$, such that

$$
\inf _{\tilde{\omega} \in B_{\omega}}\left\{\left\langle\tilde{h}-U(\tilde{h}), \omega_{2,1}\right\rangle+\left\langle\tilde{g}_{t}-2 \log f_{t}+\log c_{t}, \omega \otimes i d\right\rangle-\left\langle U_{\tilde{g}_{t}}^{v}, \omega \otimes i d\right\rangle\right\} \geq \hat{K}_{v, \varepsilon}(\omega)-\varepsilon .
$$

Take $\delta=\varepsilon$, apply the Chebyshev's inequality to (0.11) and use (0.10) to get

$$
\begin{aligned}
& \limsup _{n \rightarrow \infty} \frac{1}{n} \log \mathbb{P}_{f, n}\left\{M_{X} \in B_{\omega} \mid\left(L_{[n t] / n}=v_{[n t] / n, \forall t \in(0,1])}\right\}\right.
\end{aligned}
$$

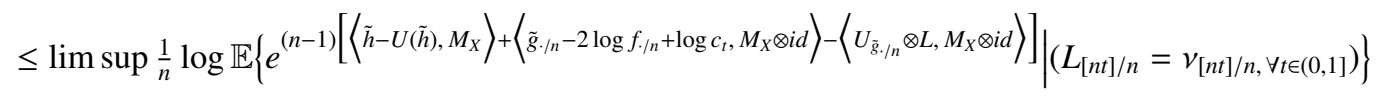

$$
\begin{aligned}
& -\hat{K}_{v, \varepsilon}(\omega)+\varepsilon \\
& \leq-\hat{K}_{v, \varepsilon}(\omega)+2 \varepsilon
\end{aligned}
$$

Using Lemma 0.4 with $\theta=\varepsilon^{-1}$ we may choose the compact set $G_{\varepsilon}$ such that

$$
\limsup _{n \rightarrow \infty} \frac{1}{n} \log \mathbb{P}_{f, n}\left\{M_{X} \notin G_{\varepsilon} \mid\left(L_{[n t] / n}=v_{[n t] / n, \forall t \in(0,1]}\right)\right\} \leq-\varepsilon^{-1} .
$$

Now, the set $F \cap G_{\varepsilon}$ is compact and therefore we may be covered by finitely many sets $B_{\omega_{1}}, \ldots, B_{\omega_{r}}$, with $\omega_{i} \in F$ , for $i=1, \ldots, r$. Hence, we have that

$$
\begin{aligned}
\mathbb{P}_{f, n}\left\{M_{X} \in F \mid L=v\right\} \leq \sum_{i=1}^{r} \mathbb{P}\{ & \left.M_{X} \in B_{\omega_{i}} \mid\left(L_{[n t] / n}=v_{[n t] / n, \forall t \in(0,1]}\right)\right\} \\
& +\mathbb{P}\left\{M_{X} \notin G_{\varepsilon} \mid\left(L_{[n t] / n}=v_{[n t] / n, \forall t \in(0,1]}\right)\right\} .
\end{aligned}
$$

Next we use (0.12) we obtain for small enough $\varepsilon>0$,

$$
\begin{aligned}
\limsup _{n \rightarrow \infty} & \frac{1}{n} \log \mathbb{P}_{f, n}\left\{M_{X} \in F \mid\left(L_{[n t] / n}=v_{[n t] / n, \forall t \in(0,1]}\right)\right\} \\
& \left.\leq \max _{i=1}^{r} \limsup _{n \rightarrow \infty} \frac{1}{n} \log \mathbb{P}_{f, n}\left|M_{X} \in B_{\omega_{i}}\right|\left(L_{[n t] / n}=v_{[n t] / n, \forall t \in(0,1]}\right)\right\}-\varepsilon^{-1} \leq-\hat{K}_{v, \varepsilon}(\omega)+2 \varepsilon
\end{aligned}
$$

Taking $\varepsilon \downarrow 0$ we get the desire statement.

We show that the function $\hat{K}_{v}(\omega)$ in Lemma 0.5 may be replaced by the good rate function

$$
K_{v}(\omega)=H\left(\omega_{2,1} \| \mu\right)+\sum_{a \in X} \omega_{2}(a) \int_{[0,1]} H\left(\omega(\cdot \mid a) \| \frac{c_{t}}{f_{t}(\cdot, a)} \otimes v_{t}(\cdot \mid a)\right) d t
$$

Lemma 0.6. For every $v \in \mathcal{D}_{\mathcal{M}}$ we have that $\hat{K}_{v}(\omega) \geq K_{v}(\omega)$. Moveover, the function $K_{v}$ is good rate function and lower semi-continuous on $\mathcal{M}(\mathcal{N} \times \mathcal{X})$.

Proof. Suppose $v_{1}=\omega$.Then, using the Jensen's inequality, by our assumption $(0.1)$ and the variational characterization of entropy we have

$$
H\left(\omega_{2,1} \| \mu\right)=\sup _{\tilde{h}}\left\{\int \tilde{h}\left(a_{1}\right) \omega_{2,1}\left(d a_{1}\right)-\log \int e^{\tilde{h}\left(a_{1}\right)} \mu\left(d a_{1}\right)\right\}
$$




$$
\begin{aligned}
& \sum_{a \in \mathcal{X}} \omega_{2}(a) \int_{[0,1]} H\left(\omega(\cdot \mid a) \| \frac{c_{t}}{f_{t}(\cdot,, a)} \otimes v_{t}(\cdot \mid a)\right) d t \\
& =\int_{[0,1] \quad \tilde{g}_{t}} \sup _{t}\left\{\int \tilde{g}_{t}(k, a) \omega(d k, d a)-\log \iint c_{t} \frac{e^{\tilde{g}(k, a)}}{f_{t}(k, a)} \omega_{2}(d a) v_{t}(d k \mid a)\right\} d t \\
& \leq \int_{[0,1]} \sup _{\tilde{g}}\left\{\int \tilde{g}_{t}(k, a) \omega(d k, d a)+\log c_{t}-2 \log c_{t}-\int \log \left(\int \frac{e^{\tilde{s} t(k, a)}}{f_{t}(k, a)} v_{t}(d k \mid a)\right) \omega_{2}(d a)\right\} d t \\
& =\int_{[0,1]} \sup _{\tilde{g}_{t}}\left\{\int \tilde{g}(k, a) \omega(d k, d a)+\log c_{t}-2 \log \int f_{t}(k, a) \omega(d k, d a)-\int \log \left(\int \frac{e^{\tilde{z} t(k, a)}}{f_{t}(k, a)} v_{t}(d k \mid a)\right) \omega_{2}(d a)\right\} d t \\
& \leq \int_{[0,1]} \sup _{\tilde{g}}\left\{\int \tilde{g}_{t}(k, a) \omega(d k, d a)+\log c_{t}-2 \int \log f_{t}(k, a) \omega(d k, d a)-\int \log \left\langle\frac{e^{\tilde{s} t}(\cdot \mid a)}{f_{t} \cdot(\cdot a)}, v_{t}(\cdot \mid a)\right\rangle \omega_{2}(d a)\right\} d t \\
& =\int_{[0,1]} \sup _{\tilde{g}_{t}}\left\{\int \tilde{g}_{t}(k, a) \omega(d k, d a)+\log c_{t}-2 \int \log f_{t}(k, a) \omega(d k, d a)-\int U_{\tilde{g}_{t}}(a) \omega_{2}(d a)\right\} d t \\
& =\hat{K}_{v}(\omega)
\end{aligned}
$$

Recall the definition of $K_{v}$ above and notice, mapping $\omega \rightarrow K_{v}(\omega)$ is continuous function. Moreover, for all $\alpha<\infty$, the level sets $\left\{K_{v} \leq \alpha\right\}$ are contained in the bounded set

$$
\left\{\omega \in \mathcal{M}(\mathcal{N} \times \mathcal{X}): \sum_{a \in \mathcal{X}} \omega_{2}(a) \int_{[0,1]} H\left(\omega(\cdot \mid a) \| \frac{c_{t}}{f(\cdot, a)} \otimes v_{t}^{v}(\cdot \mid a)\right) d t \leq \alpha\right\}
$$

and are therefore compact. Consequently, $K_{\nu}$ is a good rate function.

\subsection{Lower Bound}

We establish the lower bound by using the upper bound. To begin. we let $O$ be open subset of $\mathcal{M}(\mathcal{N} \times \mathcal{X})$.

\section{Lemma 0.7 .}

$$
\liminf _{n \rightarrow \infty} \frac{1}{n} \log \mathbb{P}_{f, n}\left\{M_{X} \in O \mid\left(L_{[n t] / n}=v_{[n t]] / n, \forall t \in(0,1]}\right)\right\} \geq-\inf _{\omega \in O} \hat{K}_{v}(\omega)
$$

Proof. Suppose $\omega=v_{1}$. We define the function $\tilde{g}_{t, \omega}: X \rightarrow \mathbb{R}$ by

$$
\tilde{g}_{t, \omega}(k, a)= \begin{cases}\log \frac{f_{t}(k, a) \omega(k \mid a)}{c_{t} v_{t}(k \mid a)} & \text { if } v_{t}(k \mid a)>0 \\ 0 & \text { otherwise }\end{cases}
$$

Let $B_{\omega}$ be open neighbourhood of $\omega$ such that for all $\tilde{\omega}, \in B_{\omega}$ we have that

$$
\left\langle\tilde{g}_{t, \omega}-2 \log f_{t}, \tilde{\omega}\right\rangle-\left\langle U_{\tilde{g}_{t, \omega}} \otimes v, \tilde{\omega} \otimes d t\right\rangle \geq\left\langle\tilde{g}_{t, \omega}-2 \log f_{t}, \omega\right\rangle-\left\langle U_{\tilde{g}_{t, \omega}} \otimes v, \omega \otimes d t\right\rangle-2 \varepsilon .
$$

We use $\tilde{\mathbb{P}}_{f, n}$ the law of the coloured preferential attachment graph obtained by transforming $\mathbb{P}_{f, n}$ using $\tilde{g}_{t, \omega}$. We observe that colour law in the transformed measure is $\omega_{2,1}$ and the linear weight function is

$$
\tilde{f_{t}}(k, a)=\frac{\omega(k \mid a)}{v_{t}(k \mid a)},
$$

where

$$
\begin{gathered}
\tilde{\gamma}(t, a)=\frac{\left|\sum_{k=0}^{\infty} k \omega(k \mid a)-1\right|}{\sum_{k=0}^{\infty} k^{2} v_{1}(k \mid a)-1} \\
\tilde{\beta}(t, a)=\frac{\left(\sum_{k=0}^{\infty} k^{2} v_{t}(k \mid a)-1\right)-\left|\sum_{k=0}^{\infty} k \omega(k \mid a)-1\right|}{\sum_{k=0}^{\infty} k^{2} v_{t}(k \mid a)-1}
\end{gathered}
$$


and that therefore $\tilde{\gamma}(t, a)+\tilde{\beta}(t, a)=1$. We use (0.6) to obtain

$$
\begin{aligned}
& \mathbb{P}_{f, n}\left\{M_{X} \in O,\left(L_{[n t] / n}=v_{[n t] / n, \forall t \in(0,1]}\right)\right\} \\
& \geq \tilde{\mathbb{E}}\left\{\frac{\tilde{\mathbb{P}}_{f, n}}{\mathbb{P}_{f, n}^{\prime \prime}}(X) \mathbb{1}_{\left\{M_{X} \in B_{\omega}\right\}},\left(L_{[n t] / n}=v_{[n t] / n, \forall t \in(0,1]}\right)\right\} \\
& =\tilde{\mathbb{E}}\left\{\exp \left(-(n-1)\left\langle\tilde{g}_{t, \omega}+\log c_{t}-U_{\tilde{g}_{t, \omega}}, M_{X} \otimes d t\right\rangle-(n-1)\left\langle\log \frac{1}{f_{t}^{2}}, M_{X} \otimes d t\right\rangle\right) \times \mathbb{1}_{\left\{M_{X} \in B_{\omega}\right\}}\right\} \\
& \geq \exp \left(-(n-1)\left\langle\tilde{g}_{t, \omega}+\log c_{t}-U_{\tilde{g}_{t, \omega}}, \omega \otimes d t\right\rangle+\varepsilon\right) \times \tilde{\mathbb{P}}_{f, n}\left\{M_{X} \in B_{\omega},\left(L_{[n t] / n}=v_{[n t] / n, \forall t \in(0,1]}\right)\right\} \\
& \left.\geq \exp \left(-(n-1)\left(\left\langle\tilde{g}_{t, \omega}, \omega \otimes d t\right\rangle-2\left\langle\log \left(\frac{c_{t}}{f_{t}}\right), M_{X} \otimes d t\right\rangle\right)+\varepsilon\right)\right) \times \tilde{\mathbb{P}}_{f, n}\left\{M_{X} \in B_{\omega},\left(L_{[n t] / n}=v_{[n t] / n, \forall t \in(0,1]}\right)\right\} \\
& \geq \exp \left(-(n-1)\left(\left\langle\tilde{g}_{t, \omega}, \omega \otimes d t\right\rangle+\varepsilon\right)-2 \int_{0}^{1} \log \left(1+\beta_{t} / \gamma_{t}\right) d t\right) \times \tilde{\mathbb{P}}_{f, n}\left\{M_{X} \in B_{\omega},\left(L_{[n t] / n}=v_{[n t] / n, \forall t \in(0,1]}\right)\right\},
\end{aligned}
$$

where we have used $c_{t}>1$ in the last inequality.

Therefore we have that

$$
\begin{aligned}
\liminf _{n \rightarrow \infty} \frac{1}{n} \log \mathbb{P}_{f, n}\left\{M_{X} \in O \mid\right. & \left.\left(L_{[n t] / n}=v_{[n t] / n, \forall t \in(0,1]}\right)\right\} \geq-\left\langle\tilde{g}_{t, \omega}, \omega \otimes d t\right\rangle+3 \varepsilon \\
+ & \liminf _{n \rightarrow \infty} \frac{1}{n} \log \widetilde{\mathbb{P}}_{f, n}\left\{M_{X} \in O \mid\left(L_{[n t] / n}=v_{[n t] / n, \forall t \in(0,1]}\right)\right\},
\end{aligned}
$$

where we have used (0.2) in the last inequality.

We complete the proof of the lower bound by showing that the last term in $(0.15)$ above vanishes. We shall use the upper bound with the measure $\mathbb{P}_{f, n}$ replaced by $\tilde{\mathbb{P}}_{f, n}$. Thus, by Lemma 0.5 we have that

$$
\begin{gathered}
\limsup _{n \rightarrow \infty} \frac{1}{n} \log \tilde{\mathbb{P}}_{f, n}\left\{M_{X} \in\left(B_{\omega}\right)^{c}\right\} \leq-\inf _{\tilde{\omega} \in\left(B_{\omega}\right)^{c}} \tilde{K}_{v}(\tilde{\omega}), \\
\tilde{K}_{v}(\tilde{\omega})= \begin{cases}\left.H\left(\tilde{\omega}_{2,1} \| v_{1,2}\right)\right)+\sum_{a \in X} \tilde{\omega}_{2}(a) \int_{[0,1]} H\left(\tilde{\omega}(\cdot \mid a) \| \frac{1}{f_{t}} \otimes v_{t}(\cdot \mid a)\right) d t & \text { if } \tilde{\omega}=v_{1}, \\
\infty & \text { otherwise, }\end{cases}
\end{gathered}
$$

where $A^{c}$ denotes complement of the set $A$. It therefore suffice to show that the infimum above is positive. Suppose for contradiction that there exits sequence $\tilde{\omega}_{n} \in\left(B_{\omega}\right)^{c}$ with $\tilde{K}_{v}\left(\tilde{\omega}_{n}\right) \downarrow 0$. Then, because the mapping $\tilde{\omega} \mapsto \tilde{K}_{v}(\tilde{\omega})$ is lower semi-continuous, we can construct a limit point $\tilde{\omega} \in\left(B_{\omega}\right)^{c}$ with $\tilde{K}_{v}(\tilde{\omega})=0$. This implies that $\tilde{\omega}_{2}=v_{1}=\omega_{2}$ and $\sum_{a \in X} \tilde{\omega}_{2}(a) \int_{(0,1]} H\left(\tilde{\omega}(\cdot \mid a) \| \frac{1}{\tilde{f}_{t}} \otimes v_{t}(\cdot \mid a)\right) d t=0$. Hence $\tilde{\omega}(k \mid a) \omega(k \mid a)=v_{t}(k \mid a) v_{t}(k \mid a)$, for all $k \in \mathcal{N}$, and $t \in(0,1)$ which yields $\tilde{\omega}=\omega$. This contradicts $\tilde{\omega} \in\left(B_{\omega}\right)^{c}$.

\subsection{Proof of Theorem 0.1 By Mixing}

To use the technique of mixing LDP results developed in (Biggins, 2004), we check the main criteria needed for the validity of (See, Biggins, 2004, Theorem 5(a)) in the following Lemma. We write $\Theta_{n}:=\mathcal{D}_{\mathcal{M}_{n}(\mathcal{N} \times X)}, \Theta:=\mathcal{D}_{\mathcal{M}(\mathcal{N} \times X)}$, and define

$$
\begin{gathered}
P_{f, n}\left(v_{1}\right):=\mathbb{P}\left[M_{X}=v_{1} \mid L_{\frac{[n t]}{n}}^{X}(\cdot, a)=v_{\frac{[n t]}{n}}(\cdot, a), t \in[0,1) \text { and } a \in X\right] \\
P_{n}\left(v_{\frac{[n t]}{n}}, t \in[0,1)\right):=\mathbb{P}\left\{L_{\frac{[n t]}{n}}^{X}=v_{\frac{[n t]}{n}}\right\}
\end{gathered}
$$

Then, the joint distribution of $M_{X}$ and $L^{X}$ is obtained by the mixture of $P_{f, n}$ and $P_{n}$ as follows:

$$
d \tilde{P}_{f, n}\left(v, v_{1}\right):=d P_{n}(v) d P_{f, n}\left(v_{1}\right) .
$$

Lemma 0.8. The family of distributions $(i)\left(P_{f, n}, n \in \mathbb{N}\right)(i i)\left(\tilde{P}_{f, n}, n \in \mathbb{N}\right)$ are exponentially tight.

Proof. (i) As this family distributions obey a large deviation upper bound with a good rate function $K_{v}(\omega)$, the family $\left(P_{f, n}, n \in \mathbb{N}\right)$ is exponentially tight. See, e.g. (Dembo and Zeitouni, 1998, Exercise 4.1.10(c)).

(ii) By (i) for every $\theta_{2}$ we can find $K_{\theta_{2}}$, compact subset of $\mathcal{D}_{\mathcal{M}(\mathcal{N} \times X)}$ such that, we have

$$
\limsup _{n \rightarrow \infty} \frac{1}{n} \log P_{f, n}\left(K_{\theta_{2}}^{c}\right) \leq-\theta_{2}
$$


Also by Lemma 0.4 , for every $\theta_{1}$ we can find $K_{\theta_{1}}$, compact subset of $\mathcal{M}(\mathcal{N} \times \mathcal{X})$ such that, we have

$$
\limsup _{n \rightarrow \infty} \frac{1}{n} \log P_{f, n}\left(K_{\theta_{1}}^{c}\right) \leq-\theta_{1}
$$

Take $\theta=\min \left(\theta_{1}, \theta_{2}\right)$ and define the relatively compact set $\Gamma_{\theta}$ by

$$
\Gamma_{\theta}:=\left\{\left(v_{1}, v\right) \in \mathcal{M}(\mathcal{N} \times \mathcal{X}) \times \mathcal{D}_{\mathcal{M}(\mathcal{N} \times \mathcal{X})}: v_{1} \in K_{\theta_{1}} \text { and } v \in K_{\theta_{2}}\right\} .
$$

Now, let $\delta>0$ and notice that, for sufficiently large $n$ we have that

$$
\tilde{P}_{f, n}\left(\Gamma_{\theta}^{c}\right) \leq \mathbb{P}\left\{M_{X} \in K_{\theta_{1}}^{c}\right\}+\mathbb{P}\left\{L^{X} \in K_{\theta_{2}}^{c}\right\} \leq C(\theta) e^{-n(\theta-\delta)} .
$$

Taking limit $n \rightarrow \infty$ followed by $\delta \downarrow 0$ of above inequality, yields

$$
\limsup _{n \rightarrow \infty} \frac{1}{n} \log \tilde{P}_{f, n}\left(\Gamma_{\theta}^{c}\right) \leq-\theta
$$

which proves the second part of the Lemma.

Now, as $J\left(v_{1}, v\right)$ is lower semi-continuous by the continuity of the relative entropies, and by Lemma 0.8 the families of distributions (i) $\left(P_{f, n}, n \in \mathbb{N}\right)$ (ii) $\left(\tilde{P}_{f, n}, n \in \mathbb{N}\right)$ are exponentially tight, we have that the latter obeys a large deviation principle with good rate function give by $J\left(v_{1}\right)$. (See, Biggins, 2004, Theorem 5(a)).

3.6 Proof of Theorem 0.3 We note that in case of this theorem $\gamma_{t}=\gamma, \beta_{t}=\beta$, and hence $c_{t}=c$ for all $t \in(0,1]$. Therefore, Theorem 0.2 and the contraction principle, (see Dembo and Zeitouni, 1998, Theorem 4.2.1) imply the large deviation principle for $M_{X}$ in the space $\mathcal{M}(\mathcal{N} \times \mathcal{X})$ with good rate function

$$
\begin{aligned}
\inf _{v \in \mathcal{D}_{\mathcal{M}}}\left\{\tilde{J}(\omega, v): \omega=v_{1}\right\} & =\inf _{v \in \mathcal{D}_{\mathcal{M}}}\left\{H\left(\omega_{2,1} \| \mu\right)+\sum_{a \in \mathcal{X}} \omega_{2}(a) \int_{0}^{1} H\left(\omega(\cdot \mid a) \| \frac{c}{f} \otimes v_{t}(\cdot \mid a)\right) d t: \omega=v_{1}\right\} \\
& \geq \inf _{v \in \mathcal{D}_{\mathcal{M}}}\left\{H\left(\omega_{2,1} \| \mu\right)+\sum_{a \in \mathcal{X}} \omega_{2}(a) H\left(\omega(\cdot \mid a) \| \frac{c}{f} \otimes \int_{0}^{1} v_{t}(\cdot \mid a) d t\right): \omega=v_{1}\right\} \\
& =H\left(\omega_{2,1} \| \mu\right)+\sum_{a \in \mathcal{X}} \omega_{2}(a) H\left(\omega(\cdot \mid a) \| \frac{c}{f} \otimes \hat{\omega}(\cdot \mid a)\right)=J(\omega)
\end{aligned}
$$

where in the third step, we have used the inequality

$$
v_{t}(k \mid a) \leq \int_{0}^{1} v_{t}(k \mid a) d t=\int_{0}^{1} v_{t}^{v}(k \mid a) d t=11-\sum_{i=0}^{k} v_{1}(i \mid a)=11-\sum_{i=0}^{k} \omega(i \mid a)=\hat{\omega}(k \mid a)
$$

for all $(k, a) \in \mathcal{N} \times \mathcal{X}$ and for all $t \in[0,1]$. This ends the proof this Theorem. 3.7Proof of Theorem 0.1

In the case of an preferential attachment graph, the function $c=\gamma(a)+\beta(a)$ degenerates to a constant $c=\gamma+\beta$ and $M_{X}=\mathcal{L} \in \mathcal{M}(\mathcal{N})$. Theorem 0.3 and the contraction principle imply a large deviation principle for $\mathcal{L}$ with good rate function

$$
J(\ell)=H\left(\ell \| \frac{(\gamma+\beta)}{f} \otimes \hat{\ell}\right)=I(\ell)
$$

where $\frac{(\gamma+\beta)}{f} \otimes \hat{\ell}(k)=\frac{(\gamma+\beta)}{f(k)} \hat{\ell}(k)$ and $\hat{\ell}(k)=11-\sum_{j=0}^{k} \ell(k)$.

\section{References}

Barabasi, A., \& Albert, R. (1999). Emergence of Scaling in Random Networks. Science, 286,509-512.

Biggins, J. D. (2004). Large deviations for mixtures. El. Comm. Probab., 9, 60-71.

Bryc, W., Minda, D., \& Sethuraman, S. (2009). Large deviations for the leaves in some random trees. Adv. in Appl. Probab., 41(3), 845-873. http://dx.doi.org/10.1239/aap/1253281066

Collevecchio, A., Cotar, C., \& LiCalzi, M. (2013). On a preferential attachment and generalized Polyaurn model. Ann. Appl. Probab., 23, 1219-1253. 
Choi, J., \& Sethuraman, S. (2011). Large deviations of the degree structures in P.A schemes. The annals of applied probability, 23, 722-763.

Dembo, A., \& O. Zeitouni, O. (1998). Large deviations techniques and applications. Springer, New York.

Dereich, S., \& Morters, P. (2009). Random networks with sublinear preferential attachement: Degree evolutions. Electronic Journal of Probability, 14, 1222-1267.

Doku-Amponsah, K. (2006). Large deviations and basic information theory for hierarchical and networked data structures. PhD Thesis, Bath.

Doku-Amponsah, K., Mettle, F. O., \& Ansah-Narh, T. (2014). Large deviations, Basic Information Theorem for Fitness Preferential Attachment Random Networks. International Journal of Statistics and Probability, 3(2), 101-109. http://dx.doi.org/10.5539/ijsp.v3n2p101

Doku-Amponsah, K. \& Mörters, P. (2010). Large deviation principle for empirical measures of coloured random graphs. The annals of Applied Probability, 20, 1989-2021. http://dx.doi.org/10.1214/09-AAP647

Krapivsky, P. L., Redner, S., \& Leyvraz, F. (2000). Connectivity of growing random networks. Physical review letters, 85, 4629.

Lawrence, S., \& Giles, C. L. (1998)(1999). Science, 280, 98 (1998); Nature, 400, 107 (1999).

M. Newman, M, Barabasi, A.-L., \& Watts, D. J. (2006). The structure and dynamics of networks. Princeton University Press.

Newman, M. E. J. (2003). The structure and function of complex networks. SIAM review.

Rudas, B., Toth, B., \& Valko, B. (2008). Random Trees and General Branching Processes. http://arxiv.org/abs/math/0503728

\section{Copyrights}

Copyright for this article is retained by the author(s), with first publication rights granted to the journal.

This is an open-access article distributed under the terms and conditions of the Creative Commons Attribution license (http://creativecommons.org/licenses/by/3.0/). 